\title{
Animales de experimentación y dermatología: entre la ética y la seguridad
}

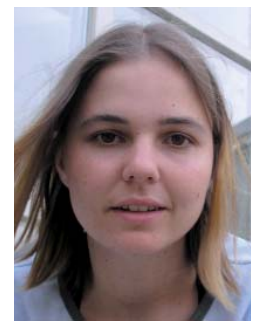

Ana d'Ors de Blas Veterinaria. Servicio de Experimentación Animal. Centro de Biología Molecular Severo Ochoa (CBMSO) Madrid

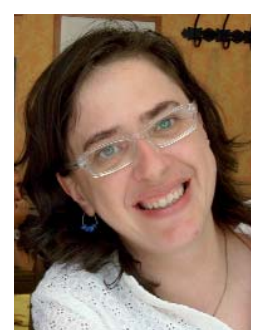

Rocío Segura Rodríguez Veterinaria.

Máster en Seguridad Alimentaria.

Directora general de Rsr.transpositiva.

\section{INTRODUCCIÓN}

La sociedad está cada vez más concienciada de que los animales sufren dolor, angustia y estrés, y de que no son meros instrumentos al servicio del ser humano y sus necesidades. Al mismo tiempo, cada día salen al mercado nuevos productos químicos (medicamentos, cosméticos, productos de limpieza, pesticidas, etc.) que pueden resultar dañinos para las personas, por lo que, antes de su utilización, deben realizarse ciertos ensayos que permitan comprobar su seguridad. Hoy por hoy, el uso de animales en algunos campos de la investigación biomédica es insustituible, y el control al que están sometidos estos ensayos es cada día mayor, no solo en los aspectos de bioseguridad y rigor, sino también en el terreno ético y humanitario. ¿Cómo podemos garantizar la seguridad de los productos que entran en contacto con nuestra piel y al mismo tiempo garantizar un trato digno a los animales que utilicemos en la investigación?

\section{EL MARCO LEGISLATIVO}

En 1986, se adoptó la Directiva Europea EU 86/609 ${ }^{1}$ para la protección de los animales utilizados en experimentación, con el objetivo de mejorar el control y establecer unos estándares mínimos sobre su alojamiento y la preparación del personal que tra- baja con ellos. A partir de este momento, se desarrollan una serie de instrumentos que buscarán mejorar el bienestar animal, como son el Tratado de Ámsterdam² de 1997, la Decisión 1999/ 575/EC de la Convención Europea para la protección de los animales vertebrados utilizados en experimentación y otros procedimientos científicos, así como la creación del Centro Europeo para la validación de métodos alternativos (ECVAM).

En el año 2002, el Parlamento Europeo revisa la Directiva EU/86/609 y, en 2008, fija las recomendaciones para su revisión, en la que participaron grupos de expertos profesionales del área académica, industria, instituciones gubernamentales y grupos profesionales de defensa de la salud y el bienestar animal.

El 20 de octubre de 2010, se publica la Directiva Europea 2010/63/EU ${ }^{4}$, que entrará en vigor como ley europea en enero de 2013. En ella, se reconoce la importancia de la utilización de animales de experimentación en los avances científicos y se refuerza la defensa y el respeto del valor intrínseco del ser animal ${ }^{5}$.

La directiva ${ }^{4}$ engloba a todos los vertebrados, a los cefalópodos (pulpos y calamares) y, en el caso de los mamíferos, también a las formas fetales. Fija todos los detalles relativos al bienestar de los animales y recoge la obligación de promover medidas que per- 
mitan la utilización completa de órganos y tejidos de los animales sacrificados. Por último, reconoce la importancia y la necesidad de algunos ensayos, e incide en la promoción de métodos alternativos y medidas que validen los datos obtenidos en otros países, para evitar la repetición de los mismos.

En España, hasta la transposición de la Directiva 2010/63/UE ${ }^{4}$, la experimentación animal está regulada por el Real Decreto $1201 / 2005^{6}$, sobre protección de los animales utilizados para experimentación y otros fines científicos, que se completa con la Ley 32/2007, de 7 de noviembre, para el cuidado de los animales, en su explotación, transporte, experimentación y sacrificio.

En relación con los productos cosméticos, el Real Decreto 209/2005 ${ }^{7}$, de 25 de febrero, sobre productos cosméticos, transfiere las normas establecidas por la directiva europea ${ }^{8}$, que obliga a las empresas del sector a utilizar sistemáticamente métodos alternativos a la experimentación animal para la evaluación de la seguridad de los productos cosméticos o de sus ingredientes o combinaciones de ingredientes.
Por lo tanto, la tendencia en la legislación europea y española es reducir el uso de los animales de experimentación, buscando modelos alternativos que permitan su sustitución, sin perjuicio del avance científico, promoviendo al mismo tiempo un uso racional y respetuoso de los mismos.

\section{USO DE LOS ANIMALES DE LABORATORIO}

El Sexto Informe ${ }^{9}$ sobre las estadísticas relativas al número de animales utilizados para experimentación y otros fines científicos en los Estados miembros de la UE pone de manifiesto que el empleo de animales de experimentación no ha sufrido grandes variaciones desde que se iniciaron estas estadísticas en el año 1996, en la Europa de los 14 miembros.

El número total de animales que se utilizó en 2008 en los 27 Estados miembros de la UE para fines experimentales y otros destinos se situó justo por encima de los 12 millones $^{9}$, de los cuales los conejos y los roedores representaron más del $80 \%$ (fig. 1) del total, y los ratones fueron, con un $59 \%$

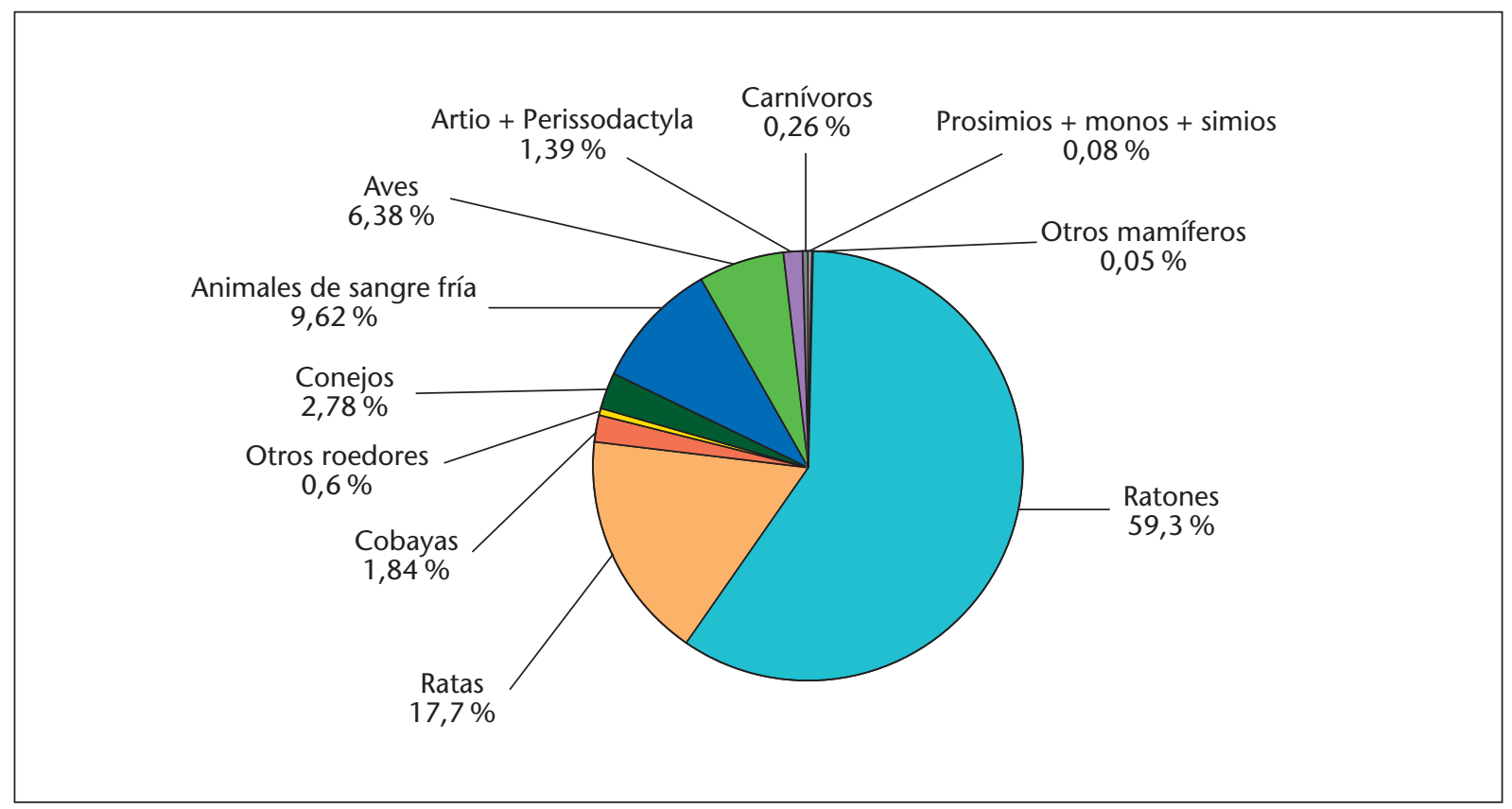

Figura 1. Porcentajes de animales utilizados por clases en los Estados miembros. 
del total, la especie más utilizada, seguida de las ratas (fig. 2). A continuación, van los vertebrados de sangre fría, reptiles, anfibios y peces $(9,6 \%)$, seguidos por las aves $(6 \%)$.

También señala que, en 2008 en la UE, no se utilizó para fines experimentales ningún gran simio, lo que ya se había detectado en los dos informes previos. Los ordenes Artiodactyla (cerdos, cabras, ovejas y bovinos) y Perissodactyla (caballos, burros y sus cruces) representaron el 1,4\% del número total de animales utilizados, mientras que el uso de carnívoros y simios no antropoides alcanzó el $0,3 \%$ y el $0,08 \%$ del total, respectivamente.

De acuerdo a lo indicado en este informe, más del $60 \%$ de los animales se utilizaron para la investigación y desarrollo en medicina, odontología y veterinaria, así como para estudios de biología fundamental, constituyendo los campos a los que se destina en la UE el mayor número de animales de laboratorio, si bien, en medicina y para odontología y veterinaria, se ha observado un drástico descenso en el número de animales utilizados (del $31 \%$ al 22,8\% o, lo que es lo mismo, de 3746028 a 2733706$)^{9}$. De ese $60 \%$, un $14,9 \%$ se empleó para el control de calidad de productos e instrumentos, y un $8,7 \%$ del total para la realización de evaluaciones de seguridad (toxicológicas y otras $)^{9}$.

Por lo tanto, el principal destino de los animales de experimentación es la realización de evaluaciones toxicológicas y otras evaluaciones de seguridad de productos o instrumentos de medicina, odontología y veterinaria (50,8\%). De este total, tan solo el 0,19\% (fig. 3) se utilizó para la evaluación toxicológica de productos/sustancias utilizados o destinados a ser utilizados principalmente como productos cosméticos o de tocador ${ }^{9}$, en el que se ha observado un descenso significativo en el número de animales utilizados (una disminución del $65 \%$ ). Este cambio radical viene motivado por la exigencia legal de eliminar progresivamente de la UE el uso de animales en las pruebas destinadas a la producción de $\operatorname{cosméticos}^{8}$.

\section{REDUCCIÓN, REFINAMIENTO Y REEMPLAZO}

En 1959, los británicos Bill Russell y Rex Burch escribieron Los principios de la técnica experimental humanitaria, en el cual se introducen por primera vez los principios de reducción, refinamiento y reemplazo, conocidos como «las $3 \mathrm{R}{ }^{10}$.

La reducción se refiere a la utilización del número mínimo de animales en los procedimientos que permita cumplir los objetivos planteados en el estudio. Esto implica una estrategia de planificación que defina claramente un objetivo razonable en base a los recursos disponibles, la elección de un modelo animal capaz de dar respuesta a las preguntas que nos planteemos, unos experimentos que utilicen un número adecuado de animales que garantice la validez de los resultados sin que se desperdicie ninguno y, por último, un correcto análisis estadístico de los resultados.

El refinamiento engloba la mejora de los métodos de estabulación, cría y mantenimiento de los

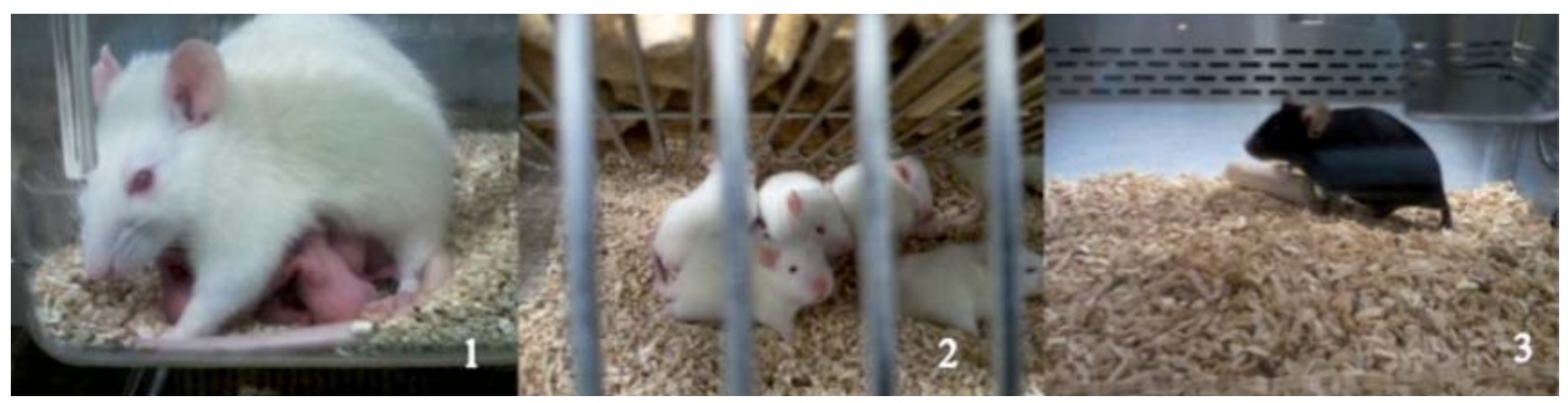

Figura 2. Animales de experimentación. 1 y 2) Ratas de la variedad Wistar. 3) Ratón de la variedad C57BI/6. 


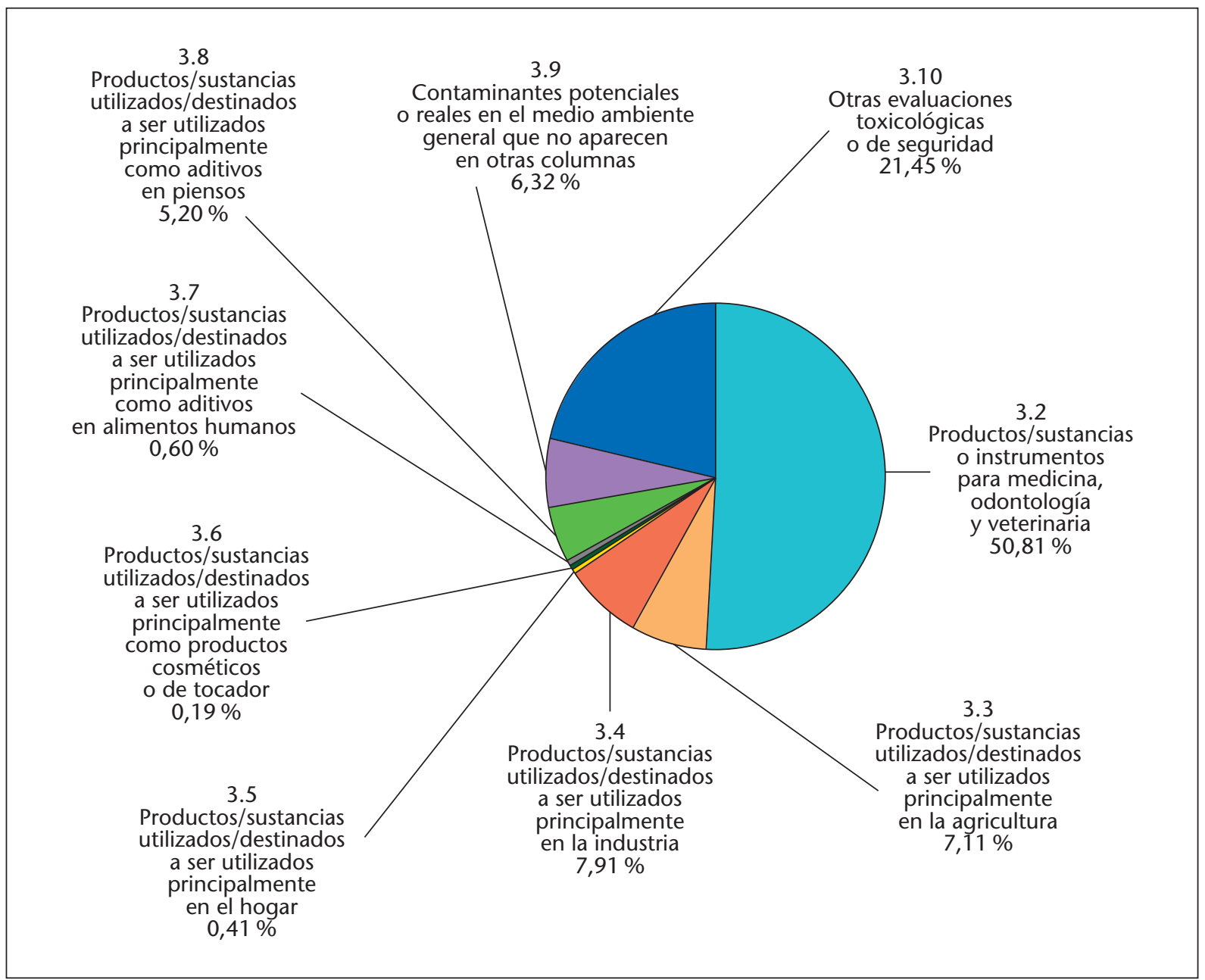

Figura 3. Número de animales utilizados en evaluaciones toxicológicas y otras evaluaciones de seguridad.

animales, así como las técnicas utilizadas en los procedimientos, eliminando o reduciendo al mínimo cualquier posible dolor, sufrimiento, angustia o daño duradero producido a los animales. Para conseguir este fin, la anestesia y analgesia deben ser indiscutibles en cualquier procedimiento quirúrgico, además de establecerse claramente unos criterios de punto final, que permitan evaluar objetivamente el sufrimiento del animal. Además, el medio ambiente del animal de laboratorio debe permitir, en la medida de lo posible, la expresión de los comportamientos naturales de la especie, no limitando la comida o bebida ni aislando individuos si no es estrictamente necesario para el procedimiento.

Finalmente, el principio de reemplazo promovería la sustitución, en los casos en los que sea posible, de los procedimientos con animales por métodos alternativos con validez científica, tales como cultivos celulares, invertebrados o modelos matemáticos ${ }^{10}$.

\section{EL SISTEMA GHS Y LA DERMATOLOGÍA}

El uso de productos químicos de síntesis para mejorar nuestra vida es una práctica internacionalmente 
extendida. Pero, además de los beneficios que nos ofrecen estos productos, debemos contar también con los efectos adversos que pueden provocar en el ser humano y en el medio ambiente. Por eso, muchos países y organizaciones han desarrollado leyes y regulaciones para el uso de estas sustancias, reflejadas en la etiqueta o en las hojas de seguridad. Sin embargo, optimizar su desarrollo y su uso a nivel mundial necesita del consenso entre los criterios de cada país, así como en el etiquetado de las sustancias.

El Globally Armonized System of Classification and Labelling of Chemicals (GHS) es un sistema desarrollado en el año 2007 por el Programa

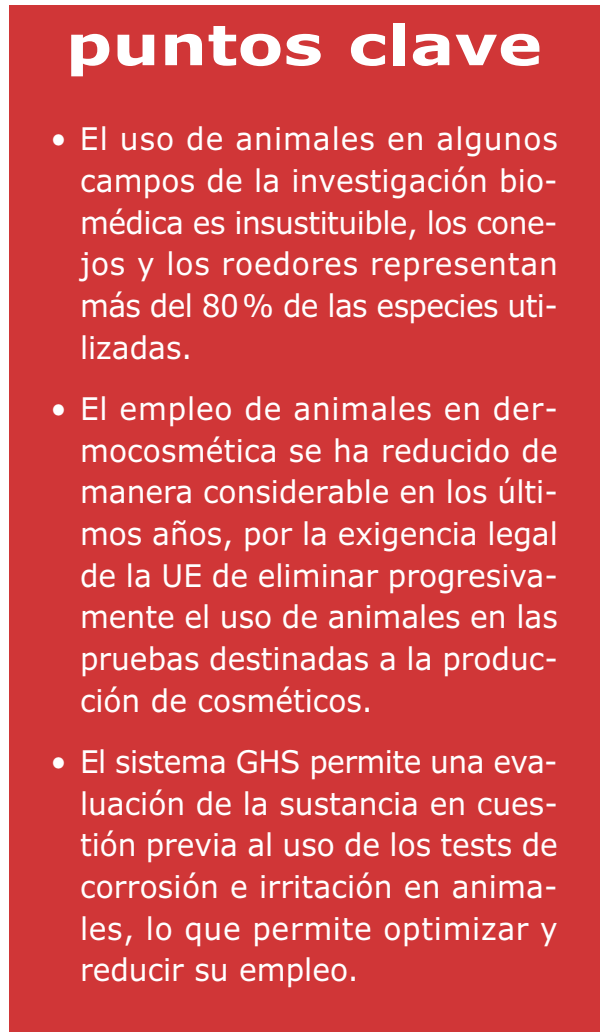

sis) de la dermis y la epidermis, también tras 4 horas de aplicación. Un sensibilizante dérmico, por su parte, se define como una sustancia que puede provocar una reacción alérgica por el contacto con la piel $^{11}$.

El sistema GHS permite una evaluación de la sustancia previa al uso del test de corrosión e irritación en animales, lo que optimiza y reduce su uso, ya que se deben valorar muchos factores antes de las pruebas directas de la sustancia sobre un organismo vivo. Reduce la severidad de los que se realizan, minimizando la gravedad de las lesiones y con ella el sufrimiento animal, ya que nunca se prueban directamente sobre los animales de Interorganización del Manejo de los Productos Químicos (IOMC) y el Grupo de Coordinación para la Armonización de los Sistemas de Clasificación de Químicos (CG/HCCS) entre otras organizaciones, cuyos objetivos son: a) mejorar la salud humana y la protección medioambiental, desarrollando un sistema internacional para la comunicación del riesgo; $b$ ) proveer de un marco regulador a aquellos países donde no existan otros sistemas o estos sean insuficientes; $c$ ) reducir la necesidad de pruebas para la evaluación de productos químicos, y $d$ ) facilitar el comercio internacional de productos químicos cuyos riesgos estén estudiados e identificados ${ }^{11}$.

El GHS incluye en su clasificación diversos daños para la salud relacionados con la piel, como son la «irritación y corrosión dérmicas», y también la «sensibilización dérmica».

La irritación se define como el daño reversible que se produce en la piel tras 4 horas de aplicación del producto testado, mientras que la corrosión se define como el daño irreversible (necro- sustancias de $\mathrm{pH}$ extremos o que hayan dado otros indicios de capacidad lesiva de la piel, por lo que solo recurrimos al ensayo directo sobre los animales si existen dudas razonables sobre su actividad. Por otro lado, recomienda un estudio previo de los datos disponibles sobre la exposición a esa sustancia (por lo que la disponibilidad de estos datos permitirá optimizar el sistema), para evitar la repetición de pruebas. Generalmente, se debe poner énfasis en la información sobre datos de personas expuestas, seguido de los tests realizados en animales y, por último, otras fuentes de información.

En algunos casos, la propia estructura química de la sustancia puede darnos pistas sobre su actividad y, en consecuencia, sobre los posibles daños que puede ocasionar. También los $\mathrm{pH}$ extremos (menores de 2 o mayores de 11,5) predicen efectos sobre la piel, si bien la capacidad tampón de algunas mezclas puede alterar este comportamiento. Además, existen sustancias cuya toxicidad sistémica está probada y es conocida, por lo que 
no procede realizar un test dérmico en el cual se exceda la dosis tóxica. En resumen, muchas veces podemos predecir la capacidad irritante o corrosiva de una sustancia sin utilizar ni un solo animal.

En cuanto a la relación de la estructura molecular de una sustancia con su actividad sobre los sistemas biológicos, son de especial interés los modelos QSAR (quantitative structure-activity relationship). Como otros modelos de regresión, son herramientas que permiten predecir el comportamiento de una variable, la molécula que se quiere analizar, basándose en los valores que tomen otras variables conocidas y su relación, la estructura molecular. La hipótesis de partida es que las estructuras similares tienen similar actividad (esto se conoce como el principio de relación estructura-actividad o SAR), pero a veces, pequeños cambios a nivel molecular pueden afectar profundamente al comportamiento de la sustancia en sistemas vivos.

Es de esperar que, en el futuro, se desarrollen nuevos sistemas que permitan predecir con mayor fiabilidad el comportamiento de las sustancias, además de otro tipo de sistemas biológicos que sustituyan en su totalidad a los ensayos con animales, encaminándonos a unas pruebas más seguras, éticas y razonables.

\section{BIBLIOGRAFÍA}

1. Council Directive $86 / 609 /$ EEC of 24 November 1986 on the approximation of laws, regulations and administrative provisions of the Member States regarding the protection of animals used for experimental and other scientific purposes.

2. Treaty of Amsterdam amending the treaty on European Union, the treaties establishing the European Communities and related acts. Protocol on protection and welfare of animals Official Journal C 340, 10 noviembre 1997.

3. 1999/575/EC: Council Decision of 23 March 1998 concerning the conclusion by the Community of the European Convention for the protection of vertebrate animals used for experimental and other scientific purposes.

4. Directiva 2010/63/UE del Parlamento Europeo y del Consejo, de 22 de septiembre de 2010, relativa a la protección de los animales utilizados para fines científicos.

5. Tremoleda JL. Comentarios sobre la Directiva Europea 2010/63/EU para la protección de animales de laboratorio. Revista de Bioética y Derecho. 2012;24:61-72.

6. BOE no 252, de 21 de octubre, p. 34367.

7. Sexto Informe sobre las estadísticas relativas al número de animales utilizados para experimentación y otros fines científicos en los Estados miembros de la Unión Europea SEC (2010) 1107. Bruselas, 8-12-2010. COM (2010) 511 final /2.

8. Directiva 76/768/CEE del Consejo, de 27 de julio de 1976.

9. Russell WMS, Burch RL. The Principles of Humane Experimental Technique. Londres: Methuen; 1959. Reprinted by UFAW, 1992.

10. OECD Guidelines for the Testing of Chemicals / OECD Series on Testing and Assessment Harmonised Integrated Classification System for Human Health and Environmental Hazards of Chemical Substances and Mixtures. Organisation for Economic Co-operation and Development, OECD 2002.

11. Wildman SA, Crippen GM. Prediction of physicochemical parameters by atomic contributions. J Chem Inf Comput. 1999; 39(5):868-73. 